\title{
Bond modelling of prestressed concrete during the prestressing force release
}

\author{
José M. Benítez · Jaime C. Gálvez
}

\begin{abstract}
This paper presents an analytical model for simulating the bond between steel and concrete, in precast prestressed concrete elements, during the prestressing force release. The model establishes a relationship between bond stress, steel and concrete stress and slip in such concrete structures. This relationship allows us to evaluate the bond stress in the transmission zone, where bond stress is not constant, along the whole prestressing force release process. The model is validated with the results of a series of tests, considering different steel indentation depths and concrete covers and is extended to evaluate the transmission length. This capability has been checked by comparing the transmission length predicted by the model and one measured experimentally in two series of tests.
\end{abstract}

Keywords Bond · Prestressed concrete ·

Transmission length $\cdot$ Modelling ·

Bond strength

\footnotetext{
J. M. Benítez

Departamento de Vehículos Aeroespaciales, E.T.S. de Ingenieros Aeronáuticos, Universidad Politécnica de Madrid, 28040 Madrid, Spain

e-mail: josemaria.benitez@upm.es

J. C. Gálvez (ه)

Departamento de Ingeniería Civil: Construcción, E.T.S. de Ingenieros de Caminos, Canales y Puertos, Universidad Politécnica de Madrid, 28040 Madrid, Spain

e-mail: jaime.galvez@upm.es; jcgalvez@caminos.upm.es
}

\section{Introduction}

Precast prestressed concrete elements are widely used for construction in Europe. Nowadays, hollow core slabs and prestressed joists are profusely used for concrete structures in building construction. In both cases, the effectiveness of prestressing force is based on the bond between steel (wire or strand) and concrete. If attention is focussed on single wire, the estimated annual production in Europe of single wire (4 and $5 \mathrm{~mm}$ diameter) for prestressed concrete exceeds 120,000 metric tons [1]. Single wire is annually used to cast more than $45 \times 10^{6} \mathrm{~m}$ of prestressed joists and about $15 \times 10^{6} \mathrm{~m}^{2}$ of hollow core slabs in Europe. The case of strands is more difficult to quantify, given that it is used for prestressed and post-tensioning concrete elements.

A frequent problem of the precast industry is to evaluate the real transmission length in precast prestressed concrete structural elements. The semiempirical formulae proposed by codes for transmission length are usually thought for conventional concrete and usual cast conditions, but high performance concrete (high-strength, self-compacting, etc.) and non-usual cast conditions ( $v . g r$. accelerated curing processes) are becoming more and more frequent. In these cases experimental measurement is needed, though the standardised methods are expensive and so difficult to apply by industry. Analytical and numerical models, based on parameters measured experimentally with tests being simpler than complete 
transmission length tests, would be welcomed. This paper deals with such an approach.

Bond between prestressed steel and concrete is essential for the success of the prestressing system. Nevertheless, bond is a complex problem and depends on many parameters. Due to its significance for practical design, it has been investigated by technical committees, e.g. see Report FIB [2], with it being an attractive challenge for many researchers, as the long list of papers on the topic corroborates.

Precast prestressed concrete structural elements are usually made as follows. The steel (strands or single wires) is prestressed in a large precast prestressing table by means of anchor heads. Such table is usually larger than $100 \mathrm{~m}$ or even $200 \mathrm{~m}$. The concrete is then cast and cured with an accelerated curing process. The prestressing force is released by approaching the anchor heads. Finally, the steel between individual structural elements is cut.

The mechanisms that contribute to bond between prestressing steel and surrounding concrete are chemical adhesion, friction and mechanical interlocking between wire indentations and concrete [3]. The geometry of the surface of the wire is very important for bond. Figure 1 shows the indention geometry of the wire, and Table 1 shows the dimensions of the indentations.

The interlock mechanism may be explained through an analogous mechanism such as that proposed by Tepfers $[4,5]$ for bars on the reinforced concrete (see Fig. 2). When the prestressing force of steel is released at the end of the element by cutting the wire, it tends to pierce in the concrete and develops tangential stress (bond stress) and radial stresses at the interface between the steel and concrete. Both stresses may be related by means of the $\alpha$ angle (see Fig. 2). The typical value of $\alpha$ for ribbed bars is $45^{\circ}$ [6], though there is no proposal for indented prestressed wires or strands.
In the case of indented wires, the wedging action generated by Poisson's effect is magnified by the indentations on the surface of the wire, increasing the tension ring [7-10]. Moreover, Hoyer's effect, presents on the ends of the prestressed concrete structural elements, is directly related with Poisson's effect [2]. Figure 3 shows a sketch of the Hoyer's effect. These aspects are beneficial for bond, though they may be harmful if concrete splits, dropping the confinement and diminishing the bond [6-8, 10-13].

Bond behaviour of reinforced concrete has been examined by many researchers, such as Gambarova and Rosati [8], Abrishami and Mitchell [9], Tepfers and Olson [5], Ogura et al. [14], Jendele and Cervenka [15] and Malvar [16] among others. Less attention has been devoted to prestressed concrete elements. In this field, bond stress versus slip curves has been studied: Gustavson [10], den Uijl [11], Abrishami and Mitchell [17] and Tassios and Bonataki [18], with a certain amount of attention being paid to the splitting action of the radial stresses and loss of confinement induced by longitudinal cracking. Several researchers have adopted an analogy between the splitting action of the reinforcement in bond and the pressure of a liquid in a pipe or sleeve [19]. These are two dimensional plane strain models, which are focussed on concrete fracture with no relationship between steel sliding and radial stresses being included, with even Poisson's effect being omitted [20, 21]. The importance of the Poisson's effect is evident, since many researchers have performed push-in tests, which take into account the increment of the wire diameter when the prestressed force is released.

This paper presents an analytical model for steelconcrete bond when the prestressing force is transmitted by releasing the steel (wire or strand). The model is based on Tepfer's proposal for reinforced concrete $[4,5]$ and on the work of van der Veen [21], who modelled the thick-walled concrete ring to
Fig. 1 Geometry of the indentations of the wire

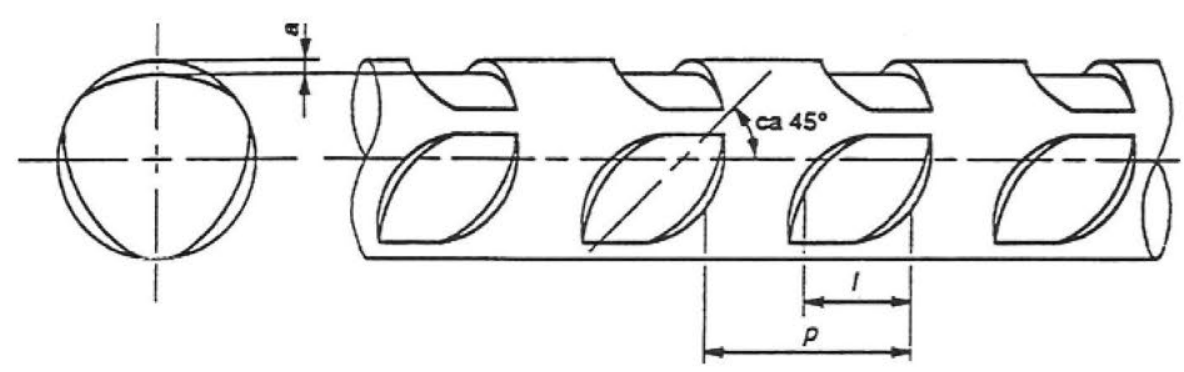


Table 1 Specified indentation dimensions, according to UNE 36094

\begin{tabular}{|c|c|c|c|c|}
\hline \multirow{3}{*}{$\begin{array}{l}\text { Nominal wire } \\
\text { diameter }\end{array}$} & \multicolumn{4}{|c|}{ Dimensions of indentations } \\
\hline & \multicolumn{2}{|c|}{ Depth $a\left(\mathrm{~mm} \times 10^{-2}\right)$} & \multirow[t]{2}{*}{ Length $l(\mathrm{~mm})$} & \multirow[t]{2}{*}{ Spacing $p(\mathrm{~mm})$} \\
\hline & T 1 & T 2 & & \\
\hline 3 & $2-6$ & & $3.5 \pm 0.5$ & $5.5 \pm 0.5$ \\
\hline 4 & $3-7$ & $5-9$ & & \\
\hline 5 & $4-8$ & $6-10$ & & \\
\hline 6 & $5-10$ & $8-13$ & $5.0 \pm 0.5$ & $8.0 \pm 0.5$ \\
\hline$\geq 7$ & $6-12$ & $10-20$ & & \\
\hline
\end{tabular}

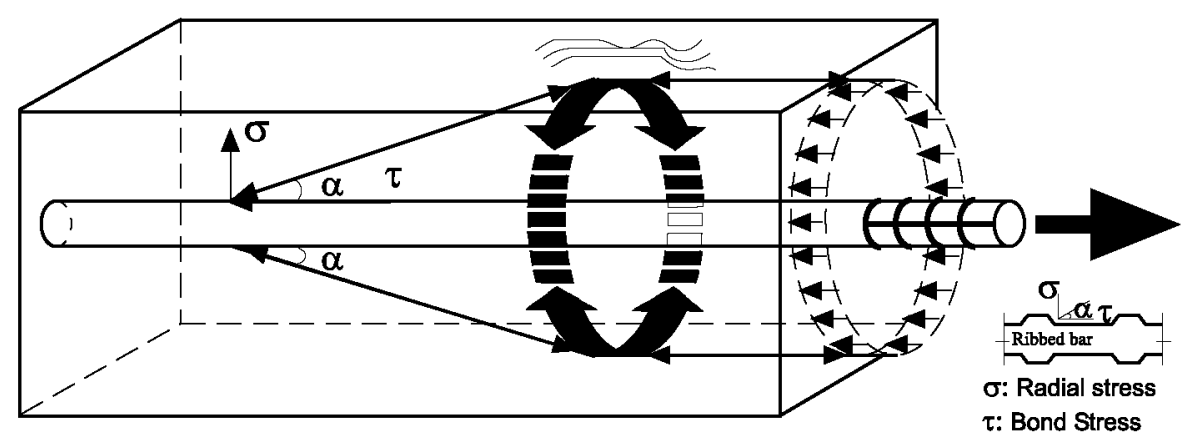

Fig. 3 Sketch of the

Hoyer's effect

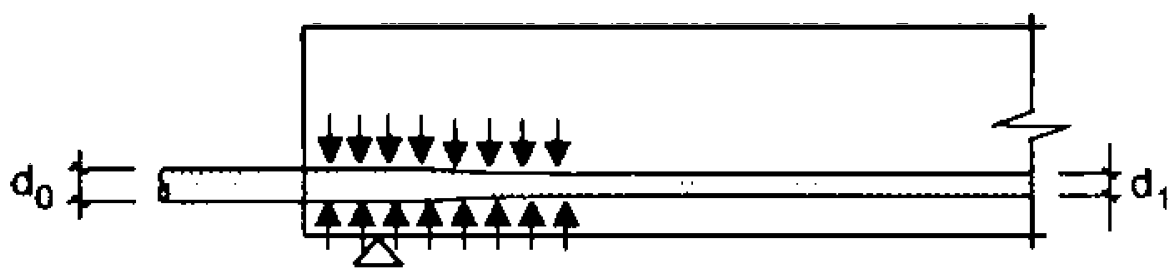

Fig. 2 Bond behaviour of deformed bars, radial component of the bond stresses balanced by tensile stresses in the uncracked ring of concrete [4] predict the cracking of the concrete on a cross-section for a known radial stress, not considering any effect of Poisson's ratio. Tepfer's model is performed for ribbed bars; it relates the normal and tangential stress between steel and concrete by an original and helpful simplified equation. On prestressed concrete, the interaction between wire and concrete is more complex than in reinforced concrete, especially in the transmission length zone, and cannot be directly modelled with the Tepfer's model, which does not take into account the Poisson's effect and the sliding between wire and concrete. The proposed analytical model allows evaluation of the radial and tangential stresses between wire and concrete, along the total length of the wire, taking into account Poisson's effect, confinement of the steel, maximum tangential stress (directly related to the depth of the indentations), concrete cover thickness and the mechanical properties of the materials. The model prediction was compared with experimental results from literature [7] and a significant degree of correspondence was achieved. The model was developed and experimentally contrasted with prestressing single wires. Crosssection of a wire has a well defined perimeter in contact with concrete and there is no helicoidally rotation when slips (this effect affects three- and seven-wire strands). Extension of the model to strands by adopting the suitable parameters does not present significant problems, especially with regard to the equivalent perimeter in contact with concrete. An application of the proposed model to evaluate the transmission length is shown in Sect. 5. The proposed model complements previous studies and models that do not include Poisson's effect and variability of the bond stress along the transmission zone.

For a practical application of the model, extension has taken place to evaluate the transmission length. The transmission length is experimentally evaluated 
in accordance with RILEM Standard [22]. This standard indicates that transmission length is the distance from the end of the specimen, where the concrete strain is equal to zero, to the section since in which the strain remains constant (see Fig. 4). Since the proposed model predicts the concrete strain along the total length of the steel (wire or strand) during prestressing force release, this is used to evaluate the transmission length. The experimental validation of the transmission length evaluation is supported by tests carried out with strands, not with wires, given that there are a greater number of experimental results with this kind of prestressed reinforcement. The model is validated with the results of two experimental series of transmission length evaluation and good agreement was reached. These results corroborate the possibility of extending the analytical model of the previous phase from wires to strands.

This work is not intended to be a substituting nor modelling of transmission length standardised testing. With this paper the authors seek to emphasise that with this analytical approach, based on simpler tests, it is possible to analyse the influence of the parameters affecting bond in the transmission length. Since the model is validated with prestressed wire specimens under careful testing, it is acknowledged that further work must be carried out to extend this modelling to majority of the prestressing concrete structural elements.

Whereas the following section examines the theoretical background, Sect. 3 studies the bond stress-slip curves calculation. Model validation is then presented and discussed in Sect. 4, with the extension to transmission length calculation being presented in

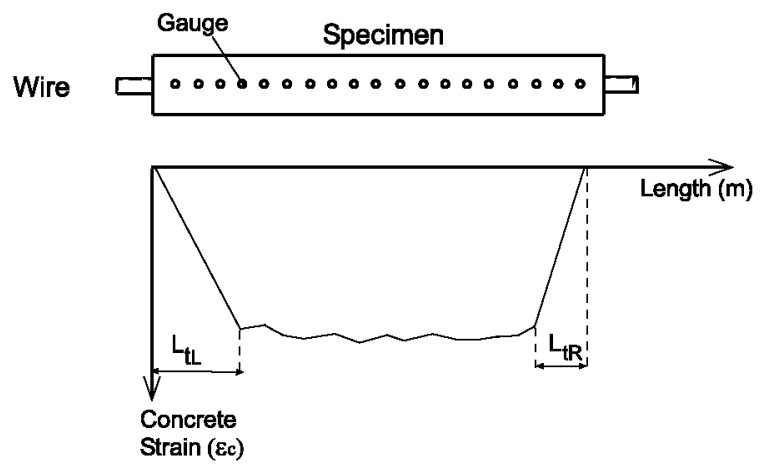

Fig. 4 Measurement of the bond length from concrete deformations [22]
Sect. 5. Finally, the conclusions obtained from the model and final comments are presented in Sect. 6.

\section{Theoretical background}

2.1 Bond between wire and concrete during release of prestressing force

As already aforementioned, the prestressing process of the precast prestressed concrete elements includes two consecutive steps: (a) steel wire prestressing, and (b) prestressing force transmission after an accelerated curing process of concrete. Bond between wire and concrete during force releasing process can be analytically expressed as follows.

Let be a prism of concrete with rectangular cross section. There is a single prestressed wire placed in the prism longitudinal axis (as Fig. 5 shows). Be $P_{0}$ the initial prestressing force in the wire. The controlled process of prestressing force release is made by means of the approaching of the wire anchorage points, in such a way that for a given instant, the force at the end of the wire is $P_{0}-\Delta P$, where $\Delta P$ is the released force. During this process, the wire tends to pierce in the concrete prism, developing tangential and normal stresses at steelconcrete interface.

It is considered a slice of the prism, with a $d x$ thickness, placed at a distance $x$ from its end as Fig. 5c shows. Equilibrium of forces in the axis prism direction (Fig. 6) leads to the following differential equation:

$\frac{\partial \Delta \sigma_{x}}{\partial x}=\frac{p_{e}}{A_{s}} \tau$

where $\Delta \sigma_{x}$ is the wire stress variation between sections $x$ and $x+d x$ (at $x=0, \Delta \sigma_{x=0}=\Delta \sigma_{0}=$ $\left.\frac{\Delta P}{A_{s}}\right), p_{e}$ is the wire perimeter, $A_{s}$ is the area of wire section and $\tau$ is the mean tangential stress between wire and concrete in the slice.

The slip between wire and concrete at any section may be expressed, based on the difference between the strains of both materials (see Fig. 6), as follows:

$\frac{d s}{d x}=\frac{\Delta \sigma_{x}}{E_{s}}-\frac{\sigma_{c}}{E_{c}}$

where $s$ is the slip between wire and concrete, $d x$ is the slice thickness, $E_{s}$ and $E_{c}$ are Young's modulus of 
Fig. 5 Manufacturing process of the prestressed concrete prism: a alone prestressed wire, b wire with cast concrete before prestressing force release, c prestressing force release (a)

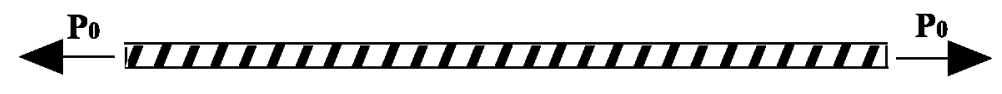

(b)

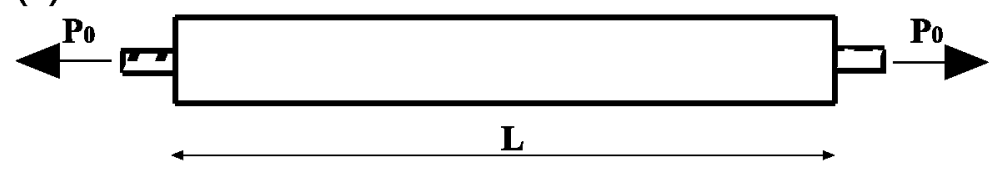

(c)

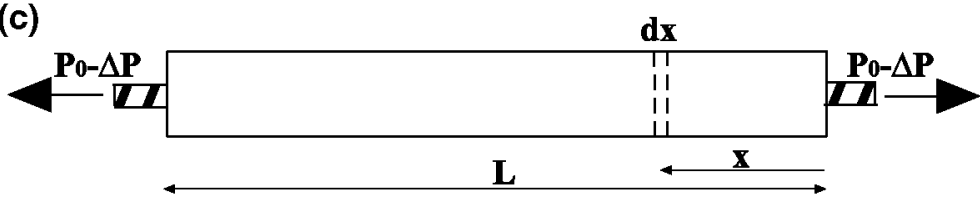

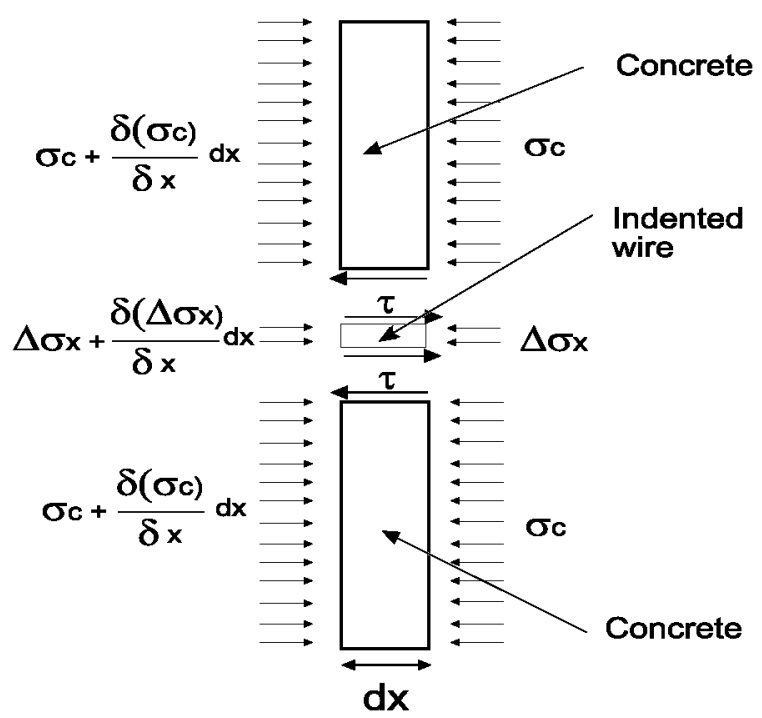

Fig. 6 Balance of stresses in a slice of $d x$ thickness

the steel and concrete, respectively, and $\sigma_{c}$ is the concrete normal stress at $x$ section.

In order to calculate the concrete stress in the section placed to an $x$ distance from the prism end, the forces equilibrium along the prism portion between this section and the prism end is established (as Fig. 7 shows). Where $\sigma_{0}$ is the wire initial stress, that is to say $P_{0} / A_{s}$, and $\Delta \sigma_{0}$, is the stress released at the wire end $(x=0)$, that is $\Delta P / A_{s}$. The following equation is obtained:

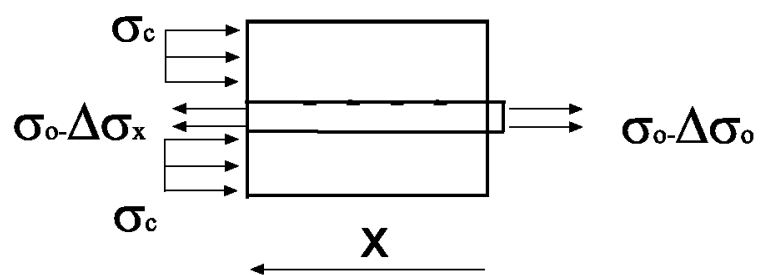

Fig. 7 Balance of forces in a part of the specimen with $x$ length, measured from the end

$\sigma_{c}=\frac{A_{s}}{A_{c}}\left(\Delta \sigma_{0}-\Delta \sigma_{x}\right)$

All the parameters have already been previously defined, except $A_{c}$, that is the net area of the concrete section (gross area minus area of wire).

$\Delta \sigma_{x}$ is found by replacing Eq. 3 into Eq. 2. Integrating Eq. 2, along $x$ distance, the slip $s$ at every section is found, as will be shown later.

\subsection{Concrete confinement of the wire}

To take into account the concrete confinement of the wire, the equilibrium of forces in the transversal direction needs to be established. Based on previous works [21] and for the sake of simplicity, the analogy of a thick-walled cylinder is adopted. Figure 8 shows the sketch of the model. $R_{1}$ is the inner radius (the radius of the wire) and $R_{2}$ is the outer radius (minimum distance from wire axis to the external 
face of the concrete prism in the transversal direction). So that $R_{2}-R_{1}$ is the minimum concrete cover of the wire in the prism.

Hereinafter, a linear elastic behaviour of the materials is adopted, with it also being assumed that there is no cracking of concrete. According to Fig. 8, $\sigma_{\theta}$ and $\sigma_{r}$ are, respectively, the circumferential and the radial stresses at any internal point of the thick-walled cylinder cross section. The circumferential strain of any point may be expressed as follows:

For steel:

$\varepsilon_{\theta s}=\frac{\sigma_{\theta s}-v_{s}\left(\sigma_{r s}+\sigma_{z s}\right)}{E_{s}}$

and for concrete:

$\varepsilon_{\theta c}=\frac{\sigma_{\theta c}-v_{c}\left(\sigma_{r c}+\sigma_{z c}\right)}{E_{c}}$

where subscript $s$ and $c$ mean steel and concrete, respectively. According to classical notation for thickwalled cylinder $\sigma_{r}$ and $\sigma_{\theta}$ are the radial and hoop stresses, and $\sigma_{z}$ the stress in normal direction to the section (axis wire direction). $v_{s}$ and $v_{c}$ are Poisson's ratios of the steel and concrete, respectively.

In this work the $\sigma_{z s}$ stress is caused by the released force $\Delta P$, and according to Fig. 8, the stress in the steel may be identified as: $\sigma_{r s}=-\sigma_{b} ; \quad \sigma_{\theta s}=-\sigma_{b} ; \quad \sigma_{z s}=-\Delta \sigma_{x}$

and in concrete:

$$
\begin{gathered}
\sigma_{r c}=-\sigma_{b} ; \quad \sigma_{\theta c}=H \sigma_{b} ; \quad \sigma_{z c}=\sigma_{c} \\
\text { with } \quad H=\frac{R_{2}^{2}+R_{1}^{2}}{R_{2}^{2}-R_{1}^{2}}
\end{gathered}
$$

where $\sigma_{b}$ is the normal stress between steel and concrete, and it is related to tangential stress $\tau$, by means of the Tepfers's equation [4]:

$\tau=\sigma_{b} \cot \alpha$

As mentioned above, there is no proposal of $\alpha$ values for indented wires and strands.

Equal strain at steel-concrete interface for both materials is assumed. Replacing Eq. 6 into Eq. 4, Eq. 7 into Eq. 5, and establishing the equality between (4) and (5), the following is obtained:

with $\left\{\begin{array}{r}\sigma_{b}=J \Delta \sigma_{x}-M \Delta \sigma_{0} \\ J=\frac{v_{s} E_{c}+\frac{A_{s}}{A_{c}} v_{c} E_{s}}{\left(1-v_{s}\right) E_{c}+\left(H+v_{c}\right) E_{s}} \\ M=\frac{v_{c} E_{s} \frac{A_{s}}{A_{c}}}{\left(1-v_{s}\right) E_{c}+\left(H+v_{c}\right) E_{s}}\end{array}\right.$

where $J$ and $M$ are dimensionless, parameters that depend only on the geometry and mechanical properties of the materials.
Fig. 8 Interaction between concrete and steel at the interface: a thick-walled cylinder approach of the prism cross-section, b stresses in concrete, c stresses in steel due to confinement (a)

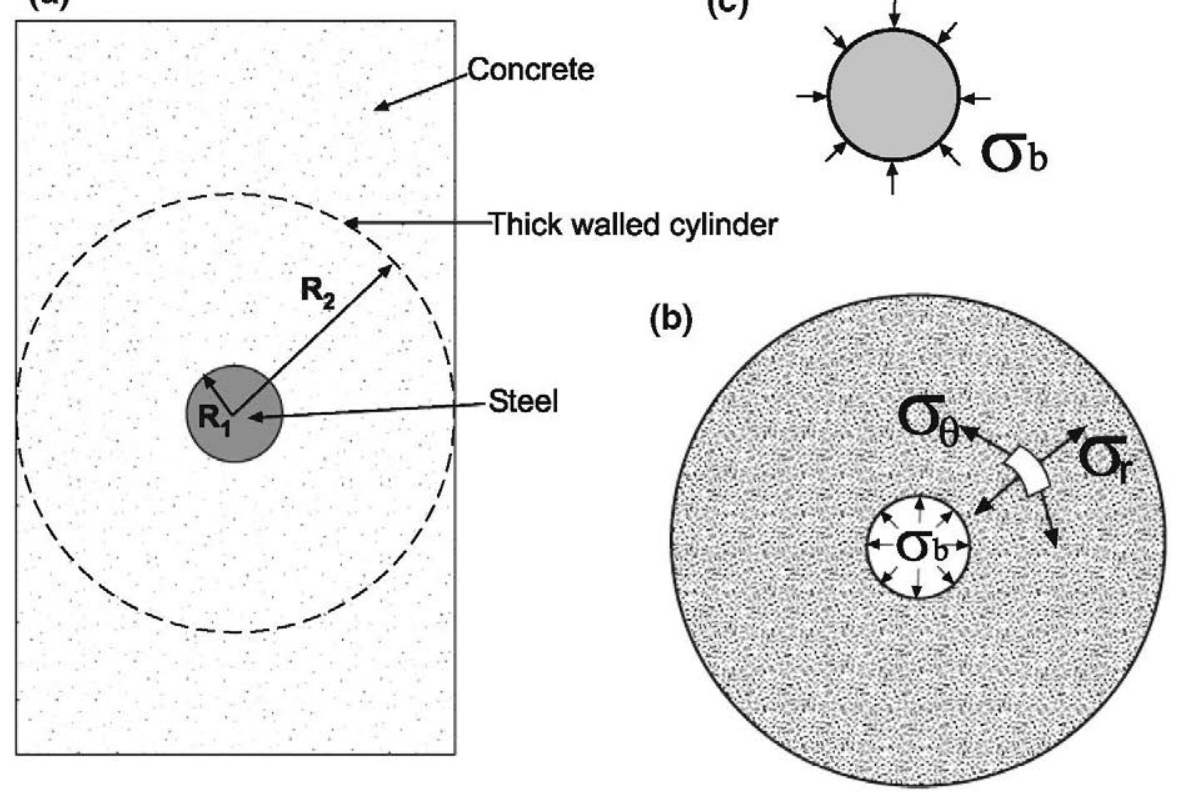


Based on the equation of Tepfers [4] that relates the tangential and normal stresses at the steelconcrete interface, the tangential stress between steel and concrete at a section placed to an $x$ distance from the prism end can be expressed:

$$
\begin{array}{r}
\tau=B_{1} \Delta \sigma_{x}-B_{2} \Delta \sigma_{0} \\
\text { with }\left\{\begin{array}{l}
B_{1}=\frac{J}{\tan \alpha} \\
B_{2}=\frac{M}{\tan \alpha}
\end{array}\right.
\end{array}
$$

where $B_{1}$ and $B_{2}$ are also dimensionless parameters that can be calculated.

Replacing Eq. 10 into Eq. 1, the following differential equation is obtained:

$$
\begin{gathered}
\frac{\partial \Delta \sigma_{x}}{\partial x}=-B \Delta \sigma_{x}+C \\
\text { with }\left\{\begin{array}{l}
B=\frac{p_{e}}{A_{s}} B_{1} \\
C=\frac{p_{e}}{A_{s}} B_{2} \Delta \sigma_{0}
\end{array}\right.
\end{gathered}
$$

Whose analytical solution is:

$$
\Delta \sigma_{x}=\frac{C}{B}+k e^{-B x}
$$

where $k$ is an integration constant to be determined for each particular case.

For every concrete prism section, the tangential stress developed between steel and concrete grows during the process of prestressing force release up to a certain value, $\tau_{\max }$, that cannot be exceeded. In this work it is assumed, for sake of simplicity, that once this critical value has been reached the tangential stress remains constant and equal to $\tau_{\max }$. Nevertheless, adoption of a lower residual value for the residual tangential stress once the maximum tangential stress has been reached, as is proposed in the Model Code [23] for bond in ribbed steel, would not present any significant difficulty.

\section{Bond stress-slip curves calculation}

To calculate $\Delta \sigma_{x}$ value, as a function of $x$, it is needed to know if the tangential stress has reached the critical value $\tau_{\max }$ at any section. In case that it happens, it is needed to know the value of $x$ along which the $\tau$ stress has exceeded the $\tau_{\max }$. This value of $x$ is noted as $X_{L I M}$. According to this aspect, integration of the Eq. 2 distinguishes two cases:

3.1 Tangential stress is lower than $\tau_{\max }$ at any value of $x\left(X_{L I M}=0\right)$

For $x=0 \Delta \sigma_{x}=\Delta \sigma_{0}$, and substituting Eq. 11 into Eq. 12, Eq. 12 may be expressed as follows:

$\Delta \sigma_{x}=\Delta \sigma_{0}\left(\frac{B_{2}}{B_{1}}+\left(1-\frac{B_{2}}{B_{1}}\right) e^{-B x}\right)$

Replacing Eq. 13 in Eq. 3 the normal stress in concrete is:

$\sigma_{c}=\frac{A_{s}}{A_{c}}\left(1-\left(\frac{B_{2}}{B_{1}}+\left(1-\frac{B_{2}}{B_{1}}\right) e^{-B x}\right)\right) \Delta \sigma_{0}$

Equations 13 and 14 allows to solve the differential equation (2).

In our particular case, a prism with an $L$ length and symmetrically released force from both ends, the integration of Eq. 2 leads to:

$$
\begin{aligned}
& s=\Delta \sigma_{0} A_{s}\left(\beta\left(\frac{B_{2}}{B_{1}} \frac{L}{2}-\frac{B_{2}-B_{1}}{B_{1}} \frac{1-e^{-\frac{B L}{2}}}{B}\right)-\frac{L}{2} \frac{1}{A_{c} E_{c}}\right) \\
& \text { with } \beta=\frac{A_{c} E_{c}+A_{s} E_{s}}{A_{c} E_{c} A_{s} E_{s}}
\end{aligned}
$$

For this prestressed concrete prism, the tangential stress between $x=0$ and $x=L / 2$ is obtained from Eq. 10 and expressed as

$\tau=\Delta \sigma_{0} B_{1}\left(1-\frac{B_{2}}{B_{1}}\right) e^{-B x}$

Obviously, $\sigma_{b}$ can be obtained from Eq. 8 .

\subsection{Tangential stress reaches $\tau_{\max }$ inside $x$ distance $\left(X_{L I M} \neq 0\right)$}

Based on the analytical solution, the $\tau$ value given by Eq. 16 may be larger than $\tau_{\max }$, the result is clearly not acceptable. To integrate the differential equation (2), a $X_{L I M}$ variable is defined, as the prism length included between the end and the section in which the tangential stress reaches the maximum value is an unknown quantity to determine.

According to the aforementioned hypothesis, the tangential stress between the steel and concrete is assumed to be constant and equal to $\tau_{\max }$ in the 
segment $0 \leq x \leq X_{L I M}$. $X_{L I M}$ is variable since it depends on the released force, growing during the process. The integration of the Eq. 1 may be easily carried out with $\tau=\tau_{\max }$ and $\Delta \sigma_{x=0}=\Delta \sigma_{0}$, obtaining the value of $\Delta \sigma_{x}$ in this segment $\left(0 \leq x \leq X_{L I M}\right)$ :

$\Delta \sigma_{x}=-\frac{p_{e}}{A_{s}} \tau_{\max } x+\Delta \sigma_{0}$

In order to determine the stresses distribution along the prism the $X_{L I M}$ value should be known. The $X_{L I M}$ value is found from Eqs. 17 and 10 producing $x=X_{L I M}$.

The first condition leads to:

$\Delta \sigma_{x=X_{L M}}=-\frac{p_{e}}{A_{s}} \tau_{\max } X_{L I M}+\Delta \sigma_{0}=\frac{C}{B} \Delta \sigma_{0}+k e^{-B X_{L M}}$

the second leads to (19) by replacing Eq. 12 in Eq. 10 and particularized for $x=X_{L I M}$

$\tau_{\max }=B_{1} k e^{-B X_{L L M}}$

giving eventually:

$X_{L I M}=\frac{A_{s}}{p_{e} \tau_{\max }}\left(\left(1-\frac{B_{2}}{B_{1}}\right) \Delta \sigma_{0}-\frac{\tau_{\max }}{B_{1}}\right)$

$k=\frac{\tau_{\max }}{B_{1}} e^{\frac{B_{1}-B_{2}}{\tau_{\max }} \Delta \sigma_{0}-1}$

Replacing $k$ in Eqs. 12 and $10, \Delta \sigma_{x}$ and $\tau$ are expressed for $x \geq X_{L I M}$ as:

$\Delta \sigma_{x}=\frac{B_{2}}{B_{1}} \Delta \sigma_{0}+\frac{\tau_{\max }}{B_{1}} e^{\frac{B_{1}-B_{2}}{\tau_{\max }} \Delta \sigma_{0}-1-B x}$

$\tau=\tau_{\max } e^{\frac{B_{1}-B_{2}}{\tau_{\max }} \Delta \sigma_{0}-1-B x}$

To determine the slip steel-concrete along the prism, the differential equation (2) is integrated, distinguishing two zones $0 \leq x \leq X_{L I M}$ and $x \geq X_{L I M}$ obtaining:

$0 \leq x \leq X_{L I M}$

$$
\begin{aligned}
x & \geq X_{L I M} \\
s= & \left(A_{s} \Delta \sigma_{0} \frac{B_{2}}{B_{1}} \beta-\frac{A_{s} \Delta \sigma_{0}}{A_{c} E_{c}}\right)\left(\frac{L}{2}-X_{L I M}\right) \\
& -\frac{\tau_{\max } \beta A_{s}}{B_{1} B}\left(e^{\left(B_{1}-B_{2}\right) \frac{\Delta \sigma_{0}}{\tau_{\max }}} 1-\frac{B L}{2}-e^{\left(B_{1}-B_{2}\right) \frac{\Delta \sigma_{0}}{\tau_{\max }}-1-B X_{L M M}}\right)
\end{aligned}
$$

\section{Model validation}

The proposed analytical model is validated with the experimental results published by Galvez et al. [7]. This experimental work studies the influence of concrete cover and the indentation depth in the transmission of normal and tangential stresses between steel and concrete during the prestressing force release. Figure 9 shows a sketch of the test set.

The prestressing load was applied by a servocontrolled testing machine, and was measured with a $25 \mathrm{kN}$ load cell. Extensometers, of $\pm 2.5 \mathrm{~mm}$ nominal travel, were used to measure the shortening of the specimen, the longitudinal crack opening displacement, and wire-concrete slip on the upper and lower faces of the prismatic specimen (see Fig. 10 for details of the placement of the extensometers).

Three series of specimens were tested. The test procedure was based on releasing, under control displacement, the prestressing force of a single wire embedded in a concrete prism. The slip between steel and concrete at the end of the specimen was recorded throughout the whole test. Three concrete covers and three indentation depths of the wire were studied. In this work the experimental results corresponding to the larger covers were the only ones adopted, the reason being guarantee of an appropriate confinement of the wire and avoidance of radial concrete cracking.

The slip at the prism ends can be obtained from Eqs. 15, 24 and 25 as a function of the geometry and mechanical properties of the concrete and the steel, the prestressed stress release at the wire anchorages, $\Delta \sigma_{0}$, the maximum tangential stress between steel and concrete and the $\alpha$ angle that relates the normal and tangential stresses at interface.

$s=\frac{\left(\left(B_{2}-B_{1}\right) \Delta \sigma_{0}+\tau_{\max }\right)\left(\beta E_{s} A_{s}\left(\Delta \sigma_{0}\left(B_{2}-B_{1}\right)+\tau_{\max }\right)+2 \Delta \sigma_{0} B_{1}\right)}{2 E_{s} B \tau_{\max } B_{1}}$ 
Fig. 9 Sketch of the test setup from Gálvez et al. [7]

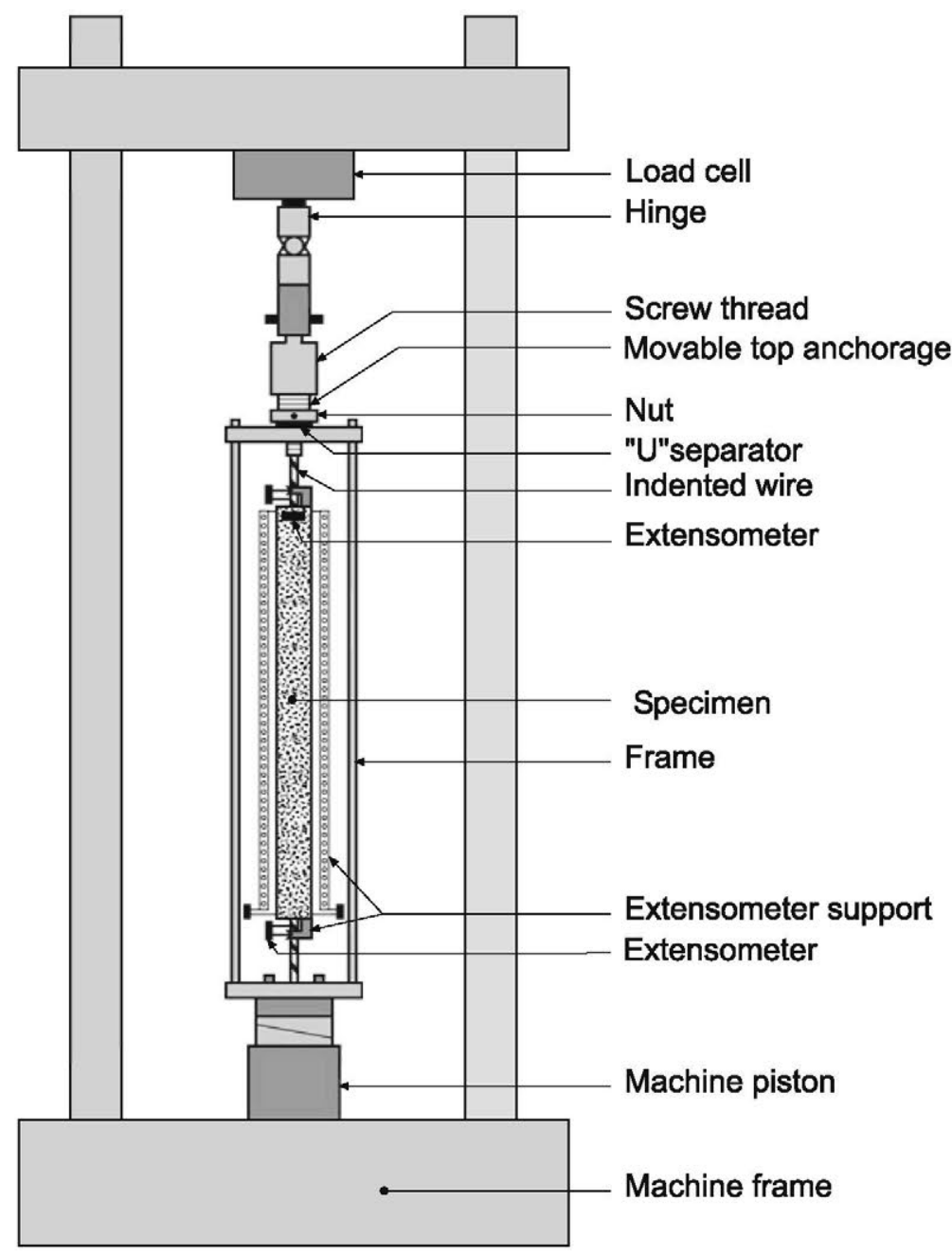

Figure 11 shows the geometry and the dimensions of the specimens. Indented wires Y $1770 \mathrm{C}$ with $4 \mathrm{~mm}$ diameter were adopted. Mechanical properties of steel were: $E=226 \mathrm{GPa}, \sigma_{0.2 \%}=1,755 \mathrm{MPa}$ and $\sigma_{u}=1,935 \mathrm{MPa}$. Table 2 shows the indentation depth of the wire. The estimated angle $\alpha$ is also included in Table 2. The maximum tangential stress at steel-concrete interface has been got experimentally [13], by means of a push-in test with small thickness specimen, and it is shown in Table 2.

Specimens manufactured with two types of covers, $2.25 \varnothing(9 \mathrm{~mm})$ and $3.25 \varnothing(13 \mathrm{~mm})$, were tested. The mechanical properties of concrete were: $E=$ $29 \mathrm{GPa}, f_{c k}=29 \mathrm{MPa}, f_{c t}=2.5 \mathrm{MPa}$ and $G_{F}=$ $102 \mathrm{~N} / \mathrm{m}$.

Figure 12 compares model prediction and experimental results of the slip between wire and concrete at the end of the specimen versus prestressed force released, for specimens with $2.25 \varnothing(9 \mathrm{~mm})$ concrete cover and, with small (Fig. 12a) and medium (Fig. 12b) indentation depths. Figure 13 shows analogous results for specimens with $3.25 \varnothing(13 \mathrm{~mm})$ concrete cover.

In the case of the specimens with smaller concrete cover (Fig. 12), the model prediction is a sufficiently accurate approximation of the experimental results, even though it tends to overvalue the slip. In the case of the specimens with larger cover (Fig. 13) the modelling fits better in the scatter band.

The most accurate model prediction of Fig. 13, specimens with larger concrete cover, is understood because larger concrete cover leads to a higher confinement of the wire, being more consistent with the hypothesis of a thick-walled cylinder for the cross-section. 

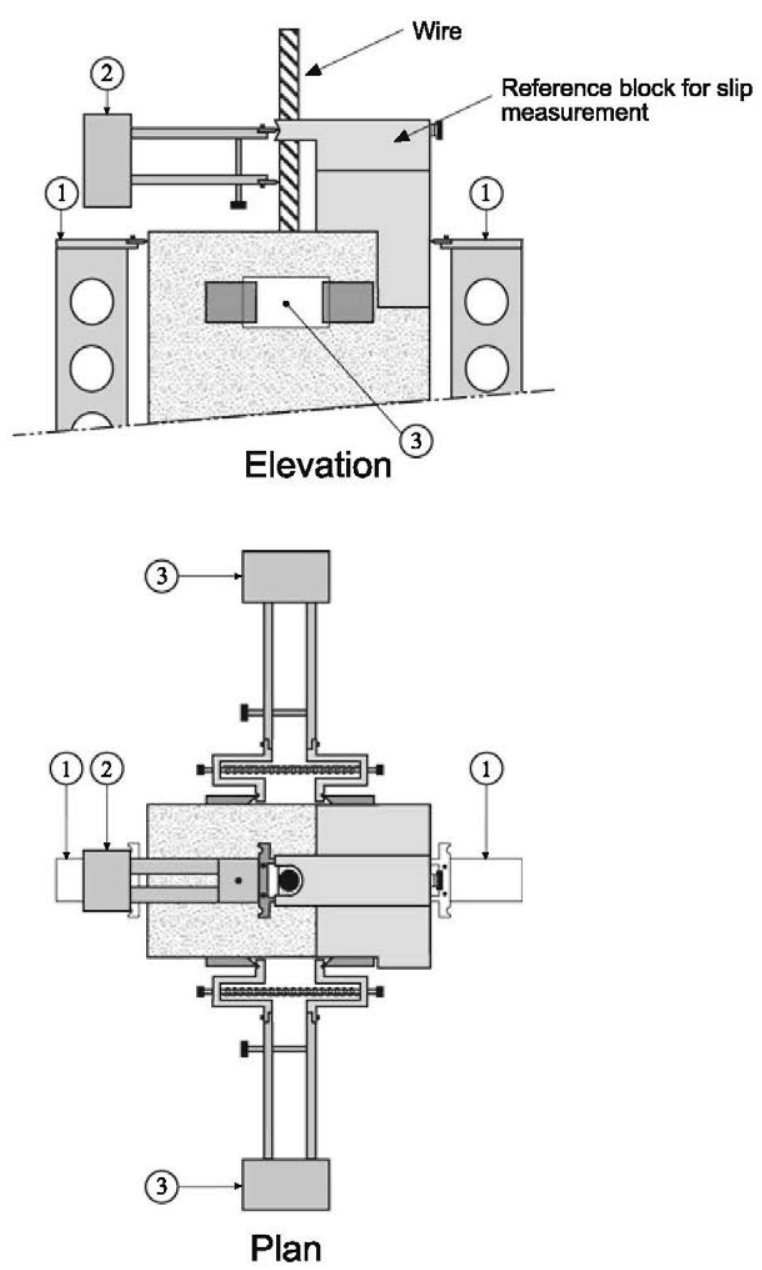

Fig. 10 Extensometers and measure devices for: (1) longitudinal shortening, (2) penetration of the wire in the upper face of the concrete prism, and (3) COD of the splitting cracks

Some authors [24] suggest that concrete cover smaller than $2.5 \varnothing$ may cause concrete splitting by a wedging action of the wire. This effect reduces the confinement of the wire. Thus, the results of Fig. 12 should be adopted with caution.

Figures 14 and 15 show the influence of the $\alpha$ angle and the Poisson's ratio of the steel, respectively, on the tangential stress along the length of the

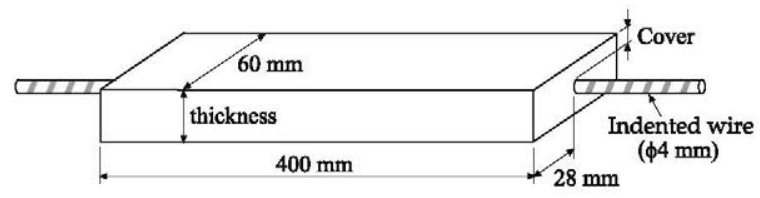

Fig. 11 Geometry and dimensions of the specimens from Gálvez et al. [7]

wire, measured from the end of the specimen (distance $x$ in Fig. 5c). Curves of both figures have been performed for the specimens with $3.25 \varnothing$ $(13 \mathrm{~mm})$ cover and medium indentation depth (maximum tangential stress $2.28 \mathrm{MPa}$ ). The influence of the $\alpha$ angle is appreciable until $x=0.031 \mathrm{~m}$. Figure 14 also shows that larger value of $\alpha$ angle corresponds with a smaller value of the tangential stress. Figure 15 shows the high influence of the Poisson's ratio on the tangential stress between wire and concrete. Even though the value of Poisson ratio is not an eligible parameter, Fig. 15 leads to the conclusion that the Poisson's ratio should be included in the bond modelling of the prestressed concrete.

\section{Transmission length calculation}

The proposed analytical model may be used to evaluate the transmission length when the concrete is not cracked and the prestressed steel (wire or strand) confined inside the concrete element.

The evaluation of the transmission length is based on the concrete strain along the specimen (see Fig. 4) which can be calculated easily by dividing the Eq. 3 by Young's Modulus of the concrete, $E_{c}$, and taking into account Eqs. 13, 17 and 22. Since the analytical model provides the strain of the concrete along the specimen, this model is used to evaluate the transmission length.

To validate the model for determining the transmission length, the results of two different experimental programs were analytically simulated.
Table 2 Indented wires indentations depth, $\alpha$ angle and maximum bond stress at interface [7, 13]

\begin{tabular}{lccc}
\hline Denomination & $\begin{array}{l}\text { Indentation } \\
\text { depth }(\mathrm{mm})\end{array}$ & $\alpha\left(^{\circ}\right)$ & $\begin{array}{l}\text { Maximum bond } \\
\text { stress (MPa) }\end{array}$ \\
\hline Smaller & $0.01-0.02$ & 80 & 2.28 \\
Medium & $0.04-0.06$ & 77 & 2.28 \\
Larger & $0.1-0.11$ & 70 & 3.16 \\
\hline
\end{tabular}



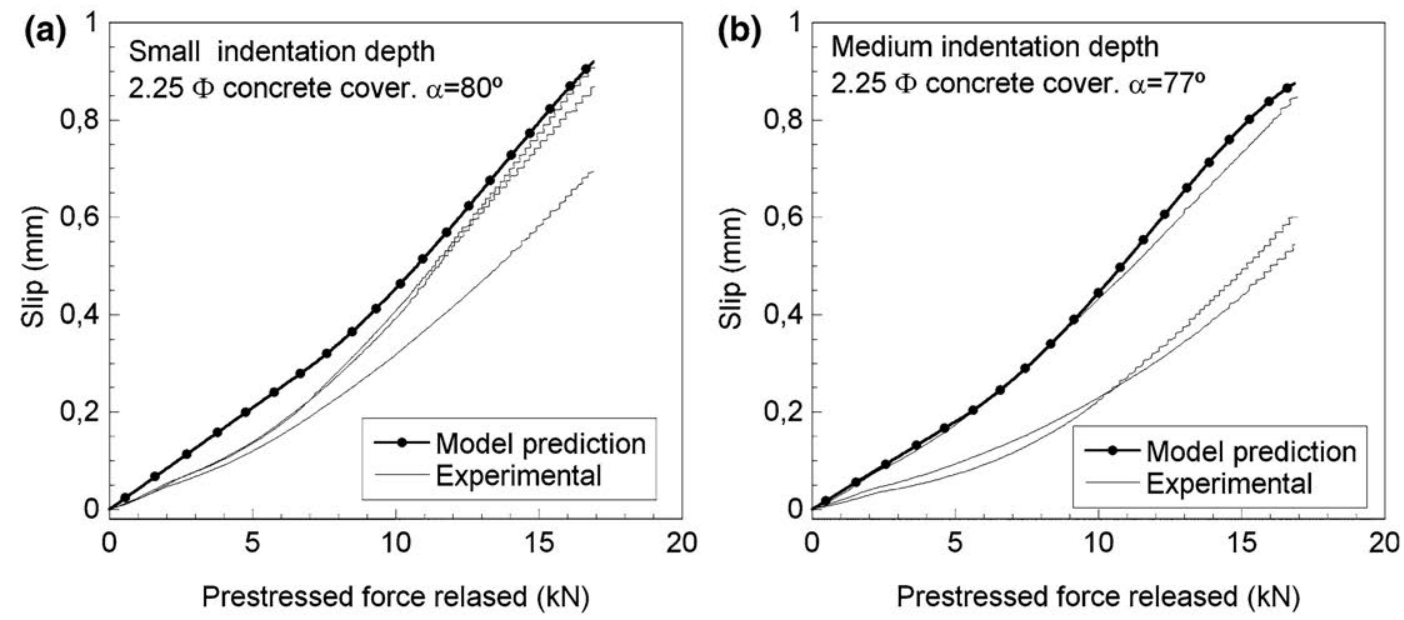

Fig. 12 Experimental results [7] and numerical prediction of the released prestressing force versus slip for specimens manufactured with $2.25 \varnothing$ concrete cover and depth indentations: a small $\left(\alpha=80^{\circ}\right)$, b medium $\left(\alpha=77^{\circ}\right)$
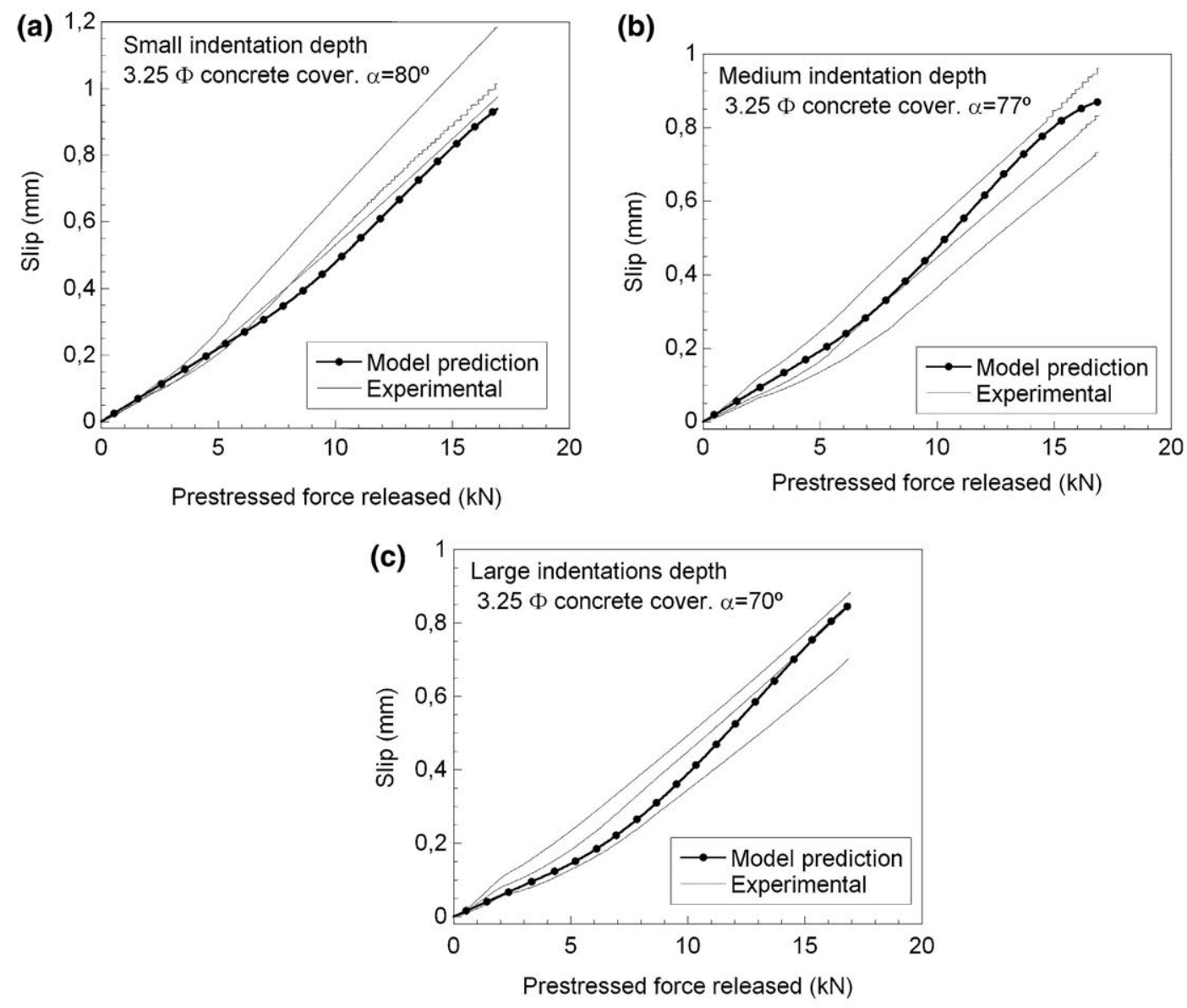

Fig. 13 Experimental results [7] and numerical prediction of the released prestressing force versus slip for specimens manufactured with $3.25 \varnothing$ concrete cover and depth indentations: a small $\left(\alpha=80^{\circ}\right)$, b medium $\left(\alpha=77^{\circ}\right)$, large $\left(\alpha=70^{\circ}\right)$ 

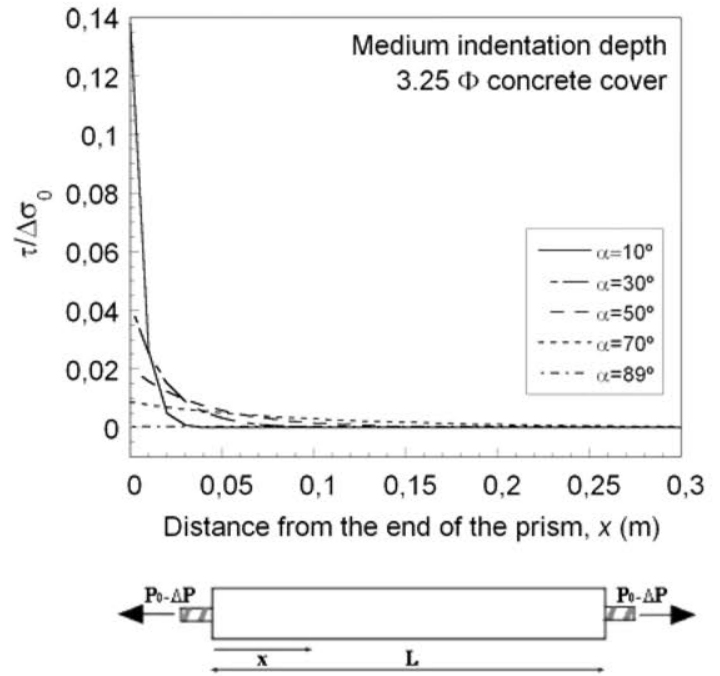

Fig. 14 Influence of the $\alpha$ angle on the tangential stress, $\tau$, between wire and concrete versus distance from the end of the prism, $x$, for specimens manufactured with $3.25 \varnothing$ concrete cover and medium depth indentations

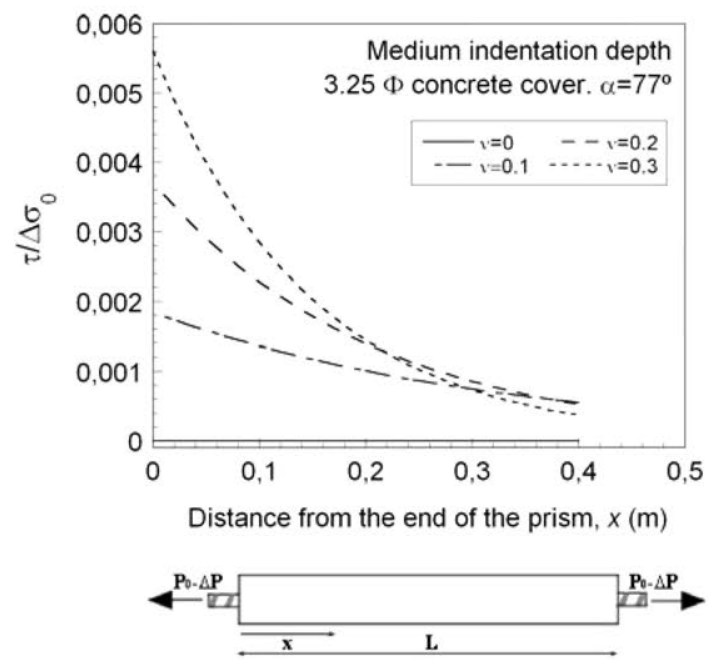

Fig. 15 Influence of Poisson's ratio of the steel on the tangential stress, $\tau$, between wire and concrete versus distance from the end of the prism, $x$, for specimens manufactured with $3.25 \varnothing$ concrete cover and medium depth indentations

The first of them was carried out by Russell and Burns [25], who manufactured pretensioned prisms of $102 \mathrm{~mm} \times 127 \mathrm{~mm} \times 3,660 \mathrm{~mm}$ with seven-wire strands. They used two different single strands: $12.7 \mathrm{~mm}$ and $15.2 \mathrm{~mm}$ strand diameter, placed in the barycentre of the cross-section. The material properties were: $E=27 \mathrm{GPa}, f_{c i}=28 \mathrm{MPa}$ and $v=0.2$, for concrete: and $E=194 \mathrm{GPa}$ and $v=0.3$, for steel.

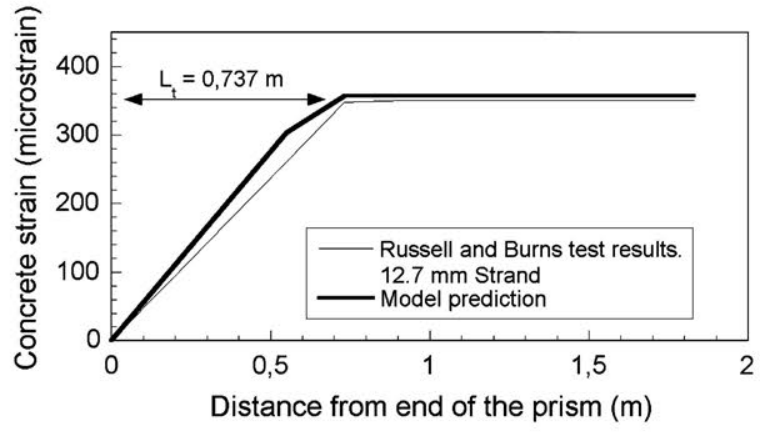

Fig. 16 Strain profile and transmission length. Comparison of model with experimental results from Russell and Burns (12.7 $\mathrm{mm}$ seven-wire strand specimens) [25]

Young's Modulus of concrete was estimated according to Model Code specifications. The force released was that corresponding with a steel stress of 1,396 MPa. With regard to the interface parameters, the critical tangential strength was $\tau_{\max }=4.0 \mathrm{MPa}$, according to the Model Code,

$\tau_{\max }=\eta_{p 1} \eta_{p 2} f_{c t d}=1.2 \times 1.0 \times 3.3=4.0 \mathrm{MPa}$

where $\eta_{p 1}$ and $\eta_{p 2}$ are parameters fixed by Model Code $\left(\eta_{p 1}\right.$ takes into account the type of prestressing tendon and $\eta_{p 2}$ the position of the tendon) and $f_{c t d}$ is the lower design tensile strength. The considered $\alpha$ angle was $\alpha=10^{\circ}$, fixed for the best fit of the numerical prediction, as will be shown later.

Figures 16 and 17 show the results for 12.7 and $15.2 \mathrm{~mm}$ strand diameters, respectively, where $L_{t}$ is the transmission length. The experimental results may be approximated by two linear branches. As figures show, the analytical model properly fit the experimental results. Concrete strains along the specimen and transmission length are accurately predicted by the proposed model.

The second series of experimental results are the tests carried out by Oh and Kim [26] who manufactured single strand pretensioned prisms of $115.2 \times$ $200 \times 3,000 \mathrm{~mm}^{3}$, with a seven-wire strand with a diameter of $12.7 \mathrm{~mm}$. In this series the wire was out of the centroid of the cross-section. Figure 18 shows the geometry and dimensions of the specimen. The only materials parameter known is the compression concrete strength at the releasing moment, $f_{c i}=$ 46.7 MPa. Other parameters have been estimated according to this data and the Model Code prescriptions, and were: $E=30 \mathrm{GPa}$ and $v=0.2$, 


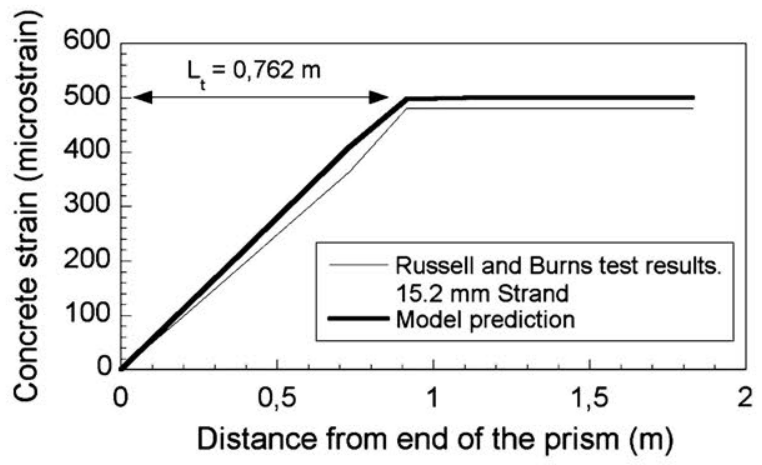

Fig. 17 Strain profile and transmission length. Comparison of model with experimental results from Russell and Burns (15.2 $\mathrm{mm}$ seven-wire strand specimens) [25]

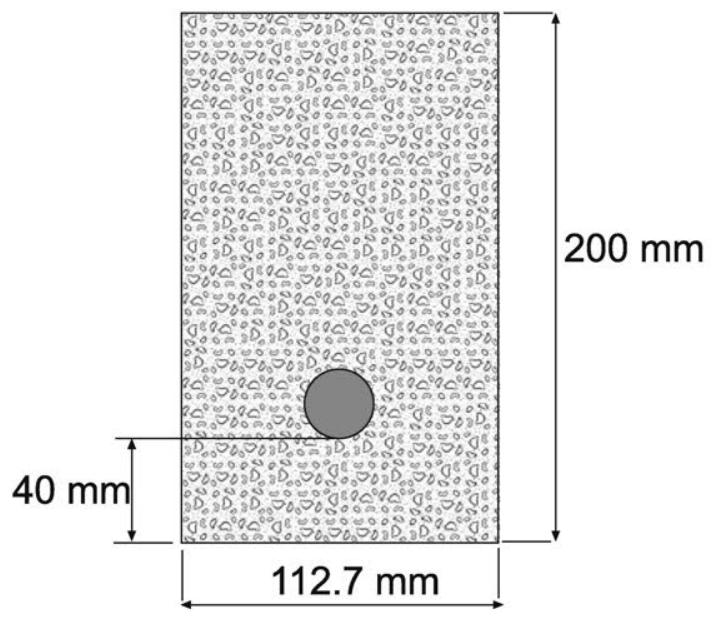

Fig. 18 Cross-section of the prisms tested by Oh and Kim [26]

for concrete; and $E=194 \mathrm{GPa}$ and $v=0.3$, for steel. The force released was that corresponding with a steel stress of $1,500 \mathrm{MPa}$, while the critical tangential stress at interface was $\tau_{\max }=6 \mathrm{MPa}$, according to the Model Code (see Eq. 26). The $\alpha$ angle adopted is $\alpha=10^{\circ}$.

Contemplating the eccentricity of the steel, Eq. 3 is modified to Eq. 27

$\sigma_{c}=\left(\Delta \sigma_{0}-\Delta \sigma_{x}\right) \frac{A_{s}}{A_{c}}+\frac{\left(\Delta \sigma_{0}-\Delta \sigma_{x}\right) A_{s} e^{2}}{I}$

where $e$ is the load eccentricity and $I$ is the crosssection moment of inertia.

Figure 19 compares the experimental results from the $\mathrm{Oh}$ and Kim test and the analytical prediction. As can be seen, the model is consistent with the transfer length and accurately predicts the concrete strain.

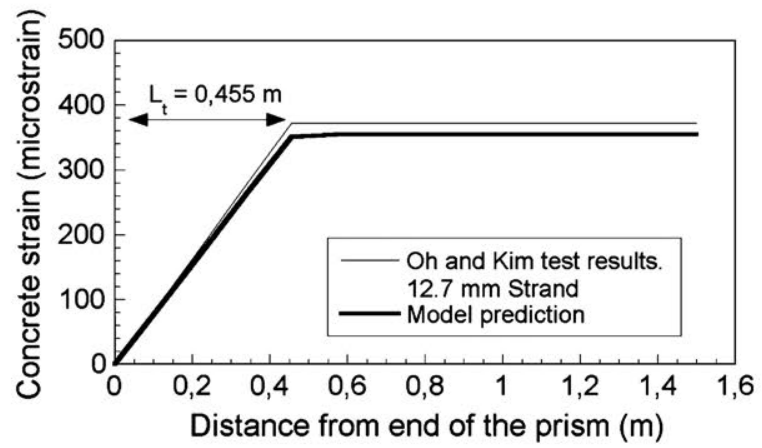

Fig. 19 Strain profile and transmission length. Comparison of model with experimental results from $\mathrm{Oh}$ and Kim tests (12.7 mm seven-wire strand specimens) [26]

Figure 20 shows the influence of the $\alpha$ angle on the tangential stress along the length of the wire, measured from the end of the specimen, for the test performed by Russell and Burns (12.7 mm sevenwire strand specimen) [25]. The influence of the $\alpha$ angle is appreciable until $x=0.04 \mathrm{~m}$.

Table 3 shows the influence of the maximum tangential stress, $\tau_{\max }$, on the estimated transmission length $L_{t}$ for the tests performed by Russell and Burns [25]. Table 3 is performed with an $\alpha$ angle of $10^{\circ}$. The best fit is reached with a maximum tangential stress of 3.0 and $4.0 \mathrm{MPa}$ for the specimens with 12.7 and $15.2 \mathrm{~mm}$ seven-wire strand, respectively. These maximum tangential stress values properly fit the estimated value from Eq. 26.

Table 4 shows the influence of the $\alpha$ angle on the estimated transmission length $L_{t}$ for tests performed by Russell and Burns [25]. Table 4 is performed with a maximum tangential stress, $\tau_{\max }$, of 3.0 and 4.0 MPa for the specimens with 12.7 and $15.2 \mathrm{~mm}$ seven-wire strand, respectively. The best fit is reached with an $\alpha$ angle of $10^{\circ}$. The influence of the $\alpha$ angle on the estimated transmission length $L_{t}$ is lower than the influence of other parameters, like maximum tangential stress.

The rotation of the strand during the sliding is not directly incorporated in the model, but is indirectly included by the fitting of the maximum tangential stress and $\alpha$ angle, affected by the strand rotation. The study of the influence of the rotation of the strand in the transmission length is beyond the scope of this paper.

The value of $\alpha$ adopted in Sect. 4 for wires $\left(70-80^{\circ}\right)$ is quite different from that used for strands $\left(10^{\circ}\right)$. It is worth noting that the $\alpha$ value has not been 


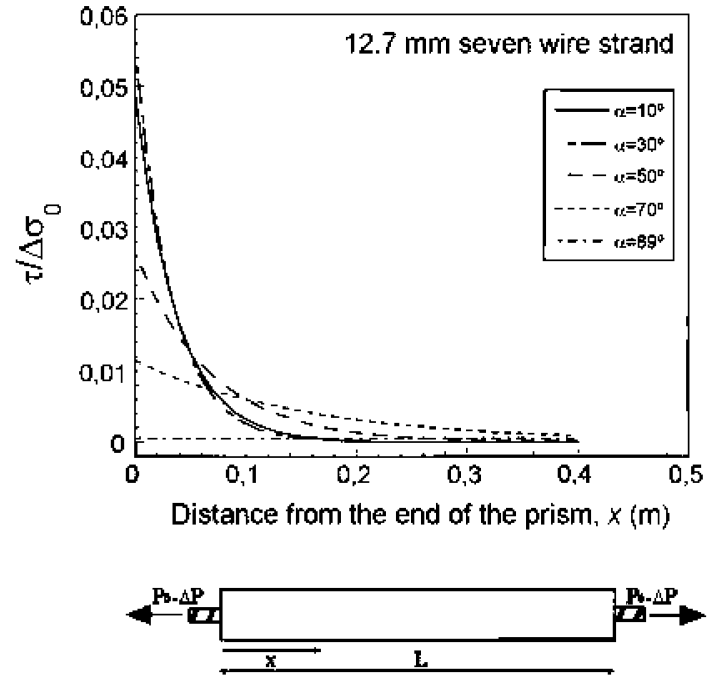

Fig. 20 Influence of the $\alpha$ angle on the tangential stress, $\tau$, between wire and concrete versus distance from the end of the prism, $x$, for specimens from Russell and Burns $(12.7 \mathrm{~mm}$ seven-wire strand specimens) [25]

Table 3 Influence of the maximum tangential stress, $\tau_{\max }$, on the estimated transmission length $L_{t}$ of the specimens from Russell and Burns [25]

\begin{tabular}{llll}
\hline$\alpha$ & $\tau_{\max }(\mathrm{MPa})$ & $\begin{array}{l}12.7 \mathrm{~mm} \text { strand } \\
L_{t}(\mathrm{~m})\end{array}$ & $\begin{array}{l}15.2 \mathrm{~mm} \text { strand } \\
L_{t}(\mathrm{~m})\end{array}$ \\
\hline $10^{\circ}$ & 1 & 2.312 & 3.438 \\
& 2 & 1.151 & 1.712 \\
& 3 & $0.764^{\mathrm{a}}$ & 1.137 \\
4 & 0.571 & $0.849^{\mathrm{a}}$ \\
\hline
\end{tabular}

${ }^{a}$ Best fit with experimental result [25]

Table 4 Influence of the $\alpha$ angle on the estimated transmission length $L_{t}$ of the specimens from Russell and Burns [25]

\begin{tabular}{lll}
\hline$\tau_{\max }$ & $\alpha$ & $L_{t}(\mathrm{~m})$ \\
\hline $3.0 \mathrm{MPa}(12.7 \mathrm{~mm}$ strand) & 10 & $0.764^{\mathrm{a}}$ \\
& 30 & 0.741 \\
& 50 & 0.706 \\
$4.0 \mathrm{MPa}$ (15.2 mm strand) & 70 & 0.618 \\
& 85 & 0.120 \\
& 10 & $0.849^{\mathrm{a}}$ \\
& 30 & 0.817 \\
& 50 & 0.768 \\
& 70 & 0.644 \\
& 85 & 0.110 \\
\hline
\end{tabular}

${ }^{\text {a }}$ Best fit with experimental result [25] experimentally measured; rather, it has been estimated based on previous tests for the case of the wires, looking for the best numerical fit in the case of the strands (see Table 4). As stated above, the rotation of the strand during sliding is not directly incorporated in the model, but is so indirectly by including the fitting of the maximum tangential stress and $\alpha$ angle, affected by the strand rotation. Moreover, the influence of the $\alpha$ angle is lower than the influence of other parameters, such as the Poisson's ratio. As Table 4 shows, the variation of the $\alpha$ angle from $10^{\circ}$ to $50^{\circ}$ leads to a variation of the transmission length in the specimens from Russell and Burns of lower than 8 per cent. Obviously, an experimental measurement of the $\alpha$ angle for wires and strands would be welcomed.

The transmission length proposed by the Model Code for a pretensioned tendon is:

$l_{b p t}=\alpha_{8} \alpha_{9} \alpha_{10} l_{b p} \frac{\sigma_{p i}}{f_{p d}}$

with

$\alpha_{8}$ gradual release 1.0, sudden release 1.25

$\alpha_{9}$ moment and shear verification 1.0, tensile stresses in anchorage zone 0.5

$\alpha_{10}$ strand 0.5 , indented wire 0.7

$l_{b p}$ basic anchorage length

$\sigma_{p i}$ steel stress after release

$f_{p d}$ design strength of tendon

As can be checked, the experimental results and the model prediction of the transmission length of the tests performed by Russell and Burns [25] and Oh and Kim [26] properly fit with the value calculated with Eq. 28.

Equation 28 leads to estimated values of the transmission length based on extensive experimental tests. Analogous equations are proposed by ACI 318 and Eurocode 2. The model proposed in this paper does not try to substitute these useful standard models, especially in the engineering design field. In many design codes the experimental approach to evaluating the transmission length is allowed and recommended, especially with non-conventional concretes and new materials, or treatments of the steel surface. In such cases, the experimental evaluation of the transmission length is expensive and requires several series of tests. The proposed model may help 
to evaluate the transmission length, or even the anchorage length, based on simpler and cheaper tests, reducing the number of transmission length tests. Moreover, the model assists in providing knowledge of the distribution of the tangential and normal stresses, between concrete and steel, in the transmission length zone.

\section{Conclusions and final comments}

An analytical model to evaluate the steel-concrete interaction in prestressed concrete elements during the prestressing force release has been proposed. The model evaluates the tangential and normal stresses in steel-concrete interface along the whole length of the element and is based on parameters with physical meaning and those that are experimentally measurable. The model has the advantage of including the Poisson ratio in the evaluation of confinement steel, as well as bond stress along the structural element. It should be noted that concrete splitting has not been taken into account. The model is suitable for confined steel in structural elements where there is no radial cracking of the concrete.

The model has been contrasted with experimental results. The slip at the ends of the prism was measured during the prestressed force release, on specimens with different concrete covers and indentation depth of the wire. In all cases, sufficiently accurate approximation of experimental results was reached.

The proposed model has been extended to evaluate the transmission length of pretensioned seven-wire strands in prestressing concrete specimens. Two series of experimental tests were used to validate the analytical modelling of the transmission length.

Based on these results, the proposed analytical model may help to the experimental evaluation of the transmission length. The relationship between bond and slip, obtained from a test simpler than complete transmission length test, may be used to estimate the transmission length. Moreover, a parametric study may be performed, based on the results of an individual transmission length test, and even different bond steel-concrete conditions may be considered in this transmission length evaluation.
Acknowledgements The authors gratefully acknowledge the financial support for the research provided by the Spanish Ministerio de Ciencia e Innovación under grant BIA-200803523 and by the Ministerio de Fomento under grants MFOM2004/9 and MFOM-01/07.

\section{References}

1. www.worldsteel.org and www.eurofer.org. Consulted on June 2008

2. FIB (2000) Bond of reinforcement in concrete. FIB bulletin 10, International Federation for Structural Concrete (fib), Laussane, Switzerland, $427 \mathrm{pp}$

3. Janney JR (1954) Nature of bond in pretensioned prestressed concrete. ACI J 25(9):717-736

4. Tepfers R (1973) A theory of bond applied to overlapped tensile reinforcement splices for deformed bars, 2 nd edn. Chalmers University of Technology, Division of Concrete Structures, Göteborg, Sweden, Publication 73:2, 328 pp

5. Tepfers R, Olsson PÅ (1992) Ring test for evaluation of bond properties of reinforcing bars. In: Bond in concrete: from research to practice, vol 1 . Riga Technical University and CEB, Riga, Latvia, pp 1-89-1-99

6. Cairns J, Johns K (1995) The splitting forces generated by bond. Mag Concr Res 47:153-165

7. Gálvez JC, Benítez JM, Tork B, Casati MJ, Cendón DA (2009) Splitting failure of precast prestressed concrete during the release of the prestressing force. Eng Fail Anal $16: 2618-2634$

8. Gambarova PG, Rosati GP (1996) Bond and splitting in reinforced concrete: test results on bar pull-out. Mater Struct 29:267-276

9. Abrishami H, Mitchell D (1992) Simulation of uniform bond stress. ACI Mater J 89(2):161-168

10. Gustavson R (2004) Experimental studies of the bond response of three-wire strands and some influencing parameters. Mater Struct 37:96-106

11. den Ujil JA (1992) Bond and splitting action of prestressed strand. In: Bond in concrete: from research to practice, vol 2. Riga Technical University and CEB, Riga, Latvia, pp 279-2-88

12. Gambarova PG, Rosati GP, Zasso B (1989) Steel-to-concrete bond after concrete splitting: constitutive laws and interface deterioration. Mater Struct 22(131):347-356

13. Benítez JM (2006) Estudio de la interacción entre el alambre preteso y el hormigón durante la transmisión de la fuerza de pretensado (in Spanish), $\mathrm{PhD}$ Thesis, Universidad Castilla La Mancha, Spain, p 219

14. Ogura N, Bolander JE, Ichinose T (2008) Analysis of bond splitting failure of deformed bars within structural concrete. Eng Struct 30(2):428-435

15. Jendele L, Cervenka J (2006) Finite element modeling of reinforcement with bond. Comput Struct 84(28):17801791

16. Malvar J (1993) Bond of reinforcement under controlled confinement. ACI Mater J 89:593-601

17. Abrishami H, Mitchell D (1993) Bond characteristics of pretensioned strand. ACI Mater J 90(3):228-235 
18. Tassios T, Bonataki E (1992) Experimental analysis of the bond behaviour of prestressed tendons. In: Bond in concrete: from research to practice, vol 1. Riga Technical University and CEB, Riga, Latvia, pp 2-29-2-37

19. Noghabai K (1999) Discrete versus smeared versus element-embebed crack models on ring problems. J Eng Mech 125(3):307-315

20. den Uijl JA, Bigaj AJ (1996) A bond model for ribbed bars based on concrete confinement. HERON 41(3):201-226

21. van der Veen C (1991) Splitting failure of reinforced concrete at various temperatures. In: van Mier JGM, Rots JG, Baker A (eds) Fracture processes in concrete, rock and ceramics. RILEM, E \& FN Spon, London, pp 941-949

22. RILEM-RPC6 (1994) Specification for the test to determine the bond properties of prestressing tendons, 1979.
RILEM Recommendations for the Testing and Use of Construction Materials, E \& FN Spon, pp 241-247

23. CEB-FIP (1990) Model code 1990, final draft. Comité Euro-International du Béton y Fédération Internationale de la Précontrainté

24. Abrishami HH, Mitchell D (1996) Influence of splitting cracks on tension stiffening. ACI Struct J 93(6):703-710

25. Russell BW, Burns NH (1997) Measurement of transfer lengths on pretensioned concrete elements. J Struct Eng 123(5):541-549

26. Oh BH, Kim ES (2001) Realistic evaluation of transfer lengths in pretensioned, prestressed concrete members. ACI Struct J 97(6):821-830 